\title{
Global Attractivity of Positive Periodic Solution for a Delayed Predator-Prey System with Diffusion and Impulses
}

\author{
Yuanfu Shao, ${ }^{1}$ Xiaolan Xie, ${ }^{2}$ and Zhixiang Ju ${ }^{1}$ \\ ${ }^{1}$ College of Science, Guilin University of Technology, Guilin, Guangxi 541004, China \\ ${ }^{2}$ Institute of Information Science and Technology, Guilin University of Technology, Guilin, Guangxi 541004, China \\ Correspondence should be addressed to Yuanfu Shao; shaoyuanfu@163.com
}

Received 5 January 2014; Revised 6 April 2014; Accepted 6 April 2014; Published 30 April 2014

Academic Editor: Zlatko Jovanoski

Copyright (c) 2014 Yuanfu Shao et al. This is an open access article distributed under the Creative Commons Attribution License, which permits unrestricted use, distribution, and reproduction in any medium, provided the original work is properly cited.

By constructing a suitable Lyapunov functional, the global attractivity of positive periodic solutions for a delayed predator-prey system with diffusion and impulses is studied in this paper. Finally, an example and numerical analysis are given to show the effectiveness of the main results.

\section{Introduction}

Recently, the existence of positive periodic solution of predator-prey system has attracted more and more attention. By using Mawhin continuation theorem and some analysis techniques, sufficient conditions of the existence of positive periodic solution are derived; see [1-3] and references cited therein.

For example, the authors in [3] proposed the following delayed periodic predator-prey system with impulses and prey diffusion in $n$-patches environments:

$$
\begin{gathered}
\dot{x}_{i}(t)=x_{i}(t)\left(r_{i}(t)-a_{i i}(t) x_{i}(t)-a_{i, n+1}(t) x_{n+1}(t)\right) \\
+\sum_{j=1, j \neq i}^{n} D_{j}(t)\left(x_{j}(t)-x_{i}(t)\right), \\
\dot{x}_{n+1}(t)=x_{n+1}(t)\left(-r_{n+1}(t)+\sum_{j=1}^{n} a_{n+1, j}(t) x_{j}\left(t-\sigma_{j}\right)\right. \\
\left.-a_{n+1, n+1}(t) x_{n+1}\left(t-\sigma_{n+1}\right)\right), \\
\Delta x_{i}\left(t_{k}\right)=x_{i}\left(t_{k}^{+}\right)-x_{i}\left(t_{k}^{-}\right)=-c_{i k} x_{i}\left(t_{k}\right), \\
t \neq t_{k},
\end{gathered}
$$

with initial conditions

$$
\begin{gathered}
x_{i}(s)=\phi_{i}(s), \\
s \in[-\sigma, 0], \quad \phi_{i}(0)>0, \quad \phi_{i} \in C\left([-\sigma, 0], R_{+}\right), \\
R_{+}=\{x \in R, x \geq 0\}, \quad i=1,2, \ldots, n+1,
\end{gathered}
$$

where $x_{i}(t)(i=1,2, \ldots, n)$ denotes the densities of prey species in patch $i$ and $x_{n+1}(t)$ denotes the density of predator. $r_{i}(t)(i=1,2, \ldots, n)$ is the intrinsic growth rates of the prey. $a_{i i}(t)(i=1,2, \ldots, n+1)$ is the density-dependent coefficients of the prey species. $a_{i, n+1}(t)$ is the capturing rates of the predator; $a_{n+1, i}(t)$ is the conversion rates of nutrients into the reproduction of predator; $i=1,2, \ldots, n . r_{n+1}(t)$ is the death rate of the predator. $D_{j}(t)(j=1,2, \ldots, n, j \neq i)$ is dispersal rate of prey species, $\sigma_{n+1} \geq 0$ denotes the delay due to negative feedback of the predator species, and $\sigma_{i}(i=1,2, \ldots, n)$ is the time delays due to gestation; that is, mature predators can only contribute to the production of predator biomass; $\sigma=\max _{1 \leq i \leq n+1}\left\{\sigma_{i}\right\}$.

By using Mawhin continuation theorem, the authors derived the sufficient conditions of the existence of positive periodic solution. However, in addition to periodic behavior, a hallmark of observed population densities is their permanent and stable behaviors [4-8]. Then, it is necessary to discuss the permanent and stable behaviors of (1) with initial conditions (2). Therefore, in this paper, we are 
devoted to the study of global attractivity of positive periodic solution for systems (1) and (2).

Throughout this paper, for $j=1,2, \ldots, n, k=1,2, \ldots$, the following conditions are assumed.

$\left(C_{1}\right) a_{i i}(t), a_{i, n+1}(t), a_{n+1, j}(t)(i=1,2, \ldots, n), r_{i}(t)(i=$ $1,2, \ldots, n+1)$, and $D_{j}(t)(j \neq i)$ are continuous, positive periodic functions with period $\omega>0$.

$\left(C_{2}\right) c_{i k}$ is positive constant satisfying $0<c_{i k}<1$ and there exists an integer $q>0$ such that

$c_{i(k+q)}=c_{i k}+\omega, \quad t_{k+q}=t_{k}+\omega$ for $i=1,2, \ldots, n+1$.

For periodic function $f$, we denote

$$
\begin{aligned}
\bar{f} & =\frac{1}{\omega} \int_{0}^{\omega} f(t) d t, \\
f^{L} & =\min _{t \in[0, \omega]}|f(t)|, \\
f^{M} & =\max _{t \in[0, \omega]}|f(t)| .
\end{aligned}
$$

The organization of this paper is as follows. In Section 2, some definitions and lemmas are introduced. In Section 3, by constructing suitable Lyapunov functional, the sufficient conditions ensuring the global attractivity of periodic solution for system (1) are established. In Section 4, an example and simulations are given to show the validity of the main results. Finally in Section 5, we conclude this paper with a brief discussion.

\section{Preliminaries}

In this section, some definitions and lemmas are introduced as follows.

Definition 1. System (1) is uniformly persistent if there exists a compact region $D \subset \operatorname{Int} R_{+}^{n+1}$ such that every solution $x(t)=$ $\left(x_{1}(t), x_{2}(t), \ldots, x_{n+1}(t)\right)^{T}$ of (1) eventually enters and remains in region $D$.

Definition 2. A bounded positive solution $x(t)=\left(x_{1}(t)\right.$, $\left.x_{2}(t), \ldots, x_{n+1}(t)\right)^{T}$ of (1) is globally asymptotically stable if for any other positive bounded solution $x^{*}(t)=$ $\left(x_{1}^{*}(t), x_{2}^{*}(t), \ldots, x_{n+1}^{*}(t)\right)^{T}$ of $(1)$, the equality $\lim _{t \rightarrow+\infty} \sum_{i=1}^{n+1}$ $\left|x_{i}(t)-x_{i}^{*}(t)\right|=0$ holds.

The following lemma ensuring the existence of positive periodic solution for system (1) is from [3].

Lemma 3 (see [3]). System (1) has at least one strictly positive $\omega$-periodic solution provided that

$\left(C_{3}\right)$

$$
\begin{aligned}
& \frac{\sum_{j=1}^{n} a_{n+1, j}^{M}\left(\bar{r}_{j}-\sum_{j=1, j \neq i}^{n} \bar{D}_{j}+(1 / \omega) \sum_{k=1}^{q} \ln \left(1-c_{j k}\right)\right)}{a_{j j}^{M}} \\
& \quad>\bar{r}_{n+1}-\frac{1}{\omega} \sum_{k=1}^{p} \ln \left(1-c_{n+1, k}\right),
\end{aligned}
$$

$$
\begin{aligned}
& \left(C_{4}\right) \\
\bar{r}_{i}- & \sum_{j=1, j \neq i}^{n} \bar{D}_{j}+\frac{1}{\omega} \sum_{k=1}^{q} \ln \left(1-c_{i k}\right) \\
>a_{i, n+1}^{M} & \frac{A \sum_{j=1}^{n} a_{n+1, j}^{M}-\bar{r}_{n+1}+(1 / \omega) \sum_{k=1}^{q} \ln \left(1-c_{n+1, k}\right)}{a_{n+1, n+1}^{L}},
\end{aligned}
$$

where $i=1,2, \ldots, n, A=\max _{1 \leq i \leq n}\left(\left(\left(r_{i}-\sum_{j=1, j \neq i}^{n} D_{j}\right)^{M}+\right.\right.$ $\left.\left.\sum_{j=1, j \neq i}^{n} D_{j}^{M}\right) /\left(a_{i i}^{L}\right)\right)$.

Lemma 4 (see [4]). If $a>0, b>0, x^{\prime}(t) \geq(\leq) x(t)(b-a x(t))$ for $t \geq 0$ and $x(0)>0$, then

$$
x(t) \geq(\leq) \frac{b}{a}\left[1+\left(\frac{b x^{-1}(0)}{a}-1\right) e^{-b t}\right]^{-1} .
$$

The following lemma is from [9]. It will be employed in establishing the asymptotic stability of (1).

Lemma 5. Let $\tau$ be a real number and let $f$ be a nonnegative function defined on $[\tau,+\infty)$ such that $f$ is integrable and uniformly continuous on $[\tau,+\infty)$; then, $\lim _{t \rightarrow \infty} f(t)=0$.

Under $\left(C_{1}\right)-\left(C_{2}\right)$, we consider the nonimpulsive delay differential equation

$$
\begin{gathered}
\dot{z}_{i}(t)=z_{i}(t)\left(r_{i}(t)-A_{i i}(t) z_{i}(t)-A_{i, n+1}(t) z_{n+1}(t)\right) \\
+\sum_{j=1, j \neq i}^{n} D_{j}(t)\left(z_{j}(t) \prod_{0<t_{k}<t}\left(1-c_{j k}\right)\right. \\
\left.\times\left(1-c_{i k}\right)^{-1}-z_{i}(t)\right), \\
i=1,2, \ldots, n, \\
\dot{z}_{n+1}(t)=z_{n+1}(t)\left(\begin{array}{c}
-r_{n+1}(t)+\sum_{j=1}^{n} A_{n+1, j}(t) z_{j}\left(t-\sigma_{j}\right) \\
\left.-A_{n+1, n+1}(t) z_{n+1}\left(t-\sigma_{n+1}\right)\right)
\end{array}\right.
\end{gathered}
$$

with initial conditions

$$
\begin{gathered}
z_{i}(s)=\phi_{i}(s), \\
s \in[-\sigma, 0], \quad \phi_{i}(0)>0, \quad \phi_{i} \in C\left([-\sigma, 0], R_{+}\right), \\
R_{+}=\{x \in R, x \geq 0\}, \quad i=1,2, \ldots, n+1,
\end{gathered}
$$

where $A_{i i}(t)=a_{i i}(t) \prod_{0<t_{k}<t}\left(1-c_{i k}\right), A_{i, n+1}(t)=a_{i, n+1}(t)$ $\prod_{0<t_{k}<t}\left(1-c_{n+1, k}\right), \quad A_{n+1, j}=a_{n+1, j} \prod_{0<t_{k}<t-\sigma_{j}}\left(1-c_{j k}\right), A_{n+1, n+1}$ $(t)=a_{n+1, n+1}(t) \prod_{0<t_{k}<t-\sigma_{n+1}}\left(1-c_{n+1, k}\right)$.

Considering (1) and (8), we have the following lemma which plays key role in the proof of the main results. The proof is similar to that of Theorem 1 in [10], and it is omitted. 
Lemma 6. Assume that $\left(C_{1}\right)-\left(C_{2}\right)$ hold. Then, one has the following.

(i) If $z(t)=\left(z_{1}(t), z_{2}(t), \ldots, z_{n+1}(t)\right)^{T}$ is a solution of (8) on $[-\sigma, \infty)$, then $x(t)=\left(x_{1}(t), x_{2}(t), \ldots, x_{n+1}(t)\right)^{T}$, $x_{i}(t)=\prod_{0<t_{k}<t}\left(1-c_{i k}\right) z_{i}(t)(i=1,2, \ldots, n+1)$ is a solution of $(1)$ on $[-\sigma, \infty)$.

(ii) If $\left(x_{1}(t), x_{2}(t), \ldots, x_{n+1}(t)\right)^{T}$ is a solution of (1) on $[-\sigma, \infty)$, then $z(t)=\left(z_{1}(t), z_{2}(t), \ldots, z_{n+1}(t)\right)^{T}$, $z_{i}(t)=\prod_{0<t_{k}<t}\left(1-c_{i k}\right)^{-1} x_{i}(t)(i=1,2, \ldots, n+1)$ is a solution of $(1)$ on $[-\sigma, \infty)$.

Lemma 7. Let $z(t)=\left(z_{1}(t), z_{2}(t), \ldots, z_{n+1}(t)\right)^{T}$ be a solution of (8); then, there exists $T>0$,

$$
0<z_{i}(t) \leq M_{i} \quad \text { for } t>T, i=1,2, \ldots, n+1,
$$

where $M_{1}=M_{2}=\cdots=M_{n}=M=$ $\max _{1 \leq i \leq n}\left\{\left(\left(r_{i}^{M}+\sum_{j=1, j \neq i}^{n} \widetilde{D}_{j}^{M}\right) /\left(A_{i i}^{L}\right)\right)+\varepsilon\right\}, M_{n+1}=\left(\left(\sum_{j=1}^{n}\right.\right.$ $\left.\left.A_{n+1, j}^{M} M\right) / A_{n+1, n+1}^{L}\right) e^{\sum_{j=1}^{n} A_{n+1, j}^{M} M \sigma_{n+1}}, \widetilde{D}_{j}(t)=D_{j}(t) \prod_{0<t_{k}<t}(1-$ $\left.c_{j k}\right)\left(1-c_{i k}\right)^{-1}$.

Proof. Define $V(t)=\max _{1 \leq i \leq n}\left\{z_{i}(t)\right\}=z_{l}(t), \iota \in[1, n]$. Calculating the upper right derivative of $V$ along the positive solution of (8), then

$$
\begin{aligned}
D^{+} V(t)= & \dot{z}_{\iota}(t) \\
= & z_{\iota}(t)\left(r_{\iota}(t)-A_{\iota}(t) z_{\iota}(t)-A_{\iota, n+1}(t) z_{n+1}(t)\right) \\
& +\sum_{j=1, j \neq \iota}^{n} D_{j}(t) \\
& \times\left(z_{j}(t) \prod_{0<t_{k}<t}\left(1-c_{j k}\right)\left(1-c_{\iota k}\right)^{-1}-z_{\iota}(t)\right) \\
\leq & z_{\iota}(t)\left(r_{\iota}^{M}-A_{\iota \iota}^{L} z_{\iota}(t)\right)+\sum_{j=1, j \neq \iota}^{n} \widetilde{D}_{j}^{M} z_{j}(t) \\
\leq & z_{\iota}(t)\left(r_{\iota}^{M}+\sum_{j=1, j \neq \iota}^{n} \widetilde{D}_{j}^{M}-A_{\iota \iota}^{L} z_{\iota}(t)\right) .
\end{aligned}
$$

By Lemma 4, for arbitrary small positive constant $\varepsilon$, there exists $T_{\imath}>0$ such that $V(t) \leq\left(\left(r_{\iota}^{M}+\sum_{j=1, j \neq \imath}^{n} \widetilde{D}_{j}^{M}\right) /\left(A_{u l}^{L}\right)\right)+\varepsilon$ for $t>T_{l}, \iota=1,2 \ldots, n$. Let $T_{1}=\max _{1 \leq l \leq n}\left\{T_{l}\right\}, M=$ $\max _{1 \leq i \leq n}\left\{\left(\left(r_{i}^{M}+\sum_{j=1, j \neq i}^{n} \widetilde{D}_{j}^{M}\right) /\left(A_{i i}^{L}\right)\right)+\varepsilon\right\}$; then, $0<z_{i}(t) \leq M$ holds for $i=1,2, \ldots, n$.
In addition, from the second equation of (8), for $t>T_{1}+$ $\widehat{\sigma}, \widehat{\sigma}=\max _{1 \leq i \leq n}\left\{\sigma_{i}\right\}$; we have

$$
\begin{aligned}
\dot{z}_{n+1}(t) \leq & z_{n+1}(t) \\
& \times\left(\sum_{j=1}^{n} A_{n+1, j}(t) z_{j}\left(t-\sigma_{j}\right)\right. \\
& \left.\quad-A_{n+1, n+1}(t) z_{n+1}\left(t-\sigma_{n+1}\right)\right) \\
\leq & z_{n+1}(t)\left(\sum_{j=1}^{n} A_{n+1, j}^{M} M-A_{n+1, n+1}^{L} z_{n+1}\left(t-\sigma_{n+1}\right)\right) .
\end{aligned}
$$

Similar argument in the proof of Lemma 2.1 of [11] shows that there exists $T_{2} \geq T_{1}+\widehat{\sigma}$ such that $z_{n+1}(t) \leq$ $\left(\left(\sum_{j=1}^{n} A_{n+1, j}^{M} M\right) /\left(A_{n+1, n+1}^{L}\right)\right) e^{\sum_{j=1}^{n} A_{n+1, j}^{M} M \sigma_{n+1}}:=M_{n+1}$ for $t>$ $T_{2}$. Take $T=T_{2}$; then, Lemma 7 follows immediately.

\section{Main Results}

In this section, by constructing suitable functional, we study the permanence and globally asymptotic stability of the periodic solution of (1).

Theorem 8. Suppose that $\left(C_{1}\right)-\left(C_{4}\right)$ hold. Further,

$\left(C_{5}\right)$

$$
-r_{n+1}^{M}+\sum_{j=1}^{n} A_{n+1, j}^{L} m>0 .
$$

Then, system (1) is uniformly persistent; that is, there exist $T^{*}>$ $T$ and $m_{i}>0$ such that

$$
m_{i} \leq z_{i}(t) \leq M_{i} \text { for } t>T^{*}, i=1,2, \ldots, n+1,
$$

where $M_{i}$ is defined by (10), $m_{1}=m_{2}=\cdots=m_{n}=m=$ $\min _{1 \leq i \leq n}\left\{\left(r_{i}^{L}-A_{i, n+1}^{M} M_{n+1}-\sum_{j=1, j \neq i}^{n} D_{j}^{M}\right) /\left(2 A_{i i}^{M}\right)\right\}, m_{n+1}<$ $\left(-r_{n+1}^{M}+m \sum_{j=1}^{n} A_{n+1, j}^{L}\right) /\left(A_{n+1, n+1}^{M}\right)$.

Proof. Define $V(t)=\min _{1 \leq i \leq n}\left\{z_{i}(t)\right\}$. Suppose that $V(t)=$ $z_{i}(t), i \in[1, n]$. Calculating the lower right derivative of $V$ along the positive solution of (8), then there exist $T_{i}>0$, for any $t>T_{i}$; we have

$$
\begin{aligned}
D_{+} V(t)= & \dot{z}_{i}(t) \\
= & z_{i}(t)\left(r_{i}(t)-A_{i i}(t) z_{i}(t)-A_{i, n+1}(t) z_{n+1}(t)\right) \\
& +\sum_{j=1}^{n} D_{j}(t)\left(z_{j}(t) \prod_{0<t_{k}<t}\left(1-c_{j k}\right)\left(1-c_{i k}\right)^{-1}\right. \\
& \left.-z_{i}(t)\right) \\
\geq & z_{i}(t)\left(r_{i}^{L}-A_{i i}^{M} z_{i}(t)-A_{i, n+1}^{M} M_{n+1}-\sum_{j=1, j \neq i}^{n} D_{j}^{M}\right) .
\end{aligned}
$$


By Lemma 4 , there exists $T_{i}^{\prime}>T_{i}>0$ such that

$z_{i}(t) \geq \frac{r_{i}^{L}-A_{i, n+1}^{M} M_{n+1}-\sum_{j=1, j \neq i}^{n} D_{j}^{M}}{A_{i i}^{M}}-\varepsilon$ for any $t>T_{i}^{\prime}$.

Equation (16) implies that there exists a positive integer $N$, for $t>T_{i}^{\prime}$; we have

$$
z_{i}(t) \geq \frac{r_{i}^{L}-A_{i, n+1}^{M} M_{n+1}-\sum_{j=1, j \neq i}^{n} D_{j}^{M}}{A_{i i}^{M}}-N \varepsilon .
$$

Noting that $\varepsilon$ is an arbitrary small positive number, we can choose $\varepsilon$ small enough such that

$$
N \varepsilon \leq \frac{r_{i}^{L}-A_{i, n+1}^{M} M_{n+1}-\sum_{j=1, j \neq i}^{n} D_{j}^{M}}{2 A_{i i}^{M}}
$$

Thus, for any $t>T_{i}^{\prime},(17)$ and (18) lead to

$$
z_{i}(t) \geq \frac{r_{i}^{L}-A_{i, n+1}^{M} M_{n+1}-\sum_{j=1, j \neq i}^{n} D_{j}^{M}}{2 A_{i i}^{M}} .
$$

Let $T^{\prime}=\max _{1 \leq i \leq n}\left\{T_{i}^{\prime}\right\}, m=\min _{1 \leq i \leq n}\left\{\left(r_{i}^{L}-A_{i, n+1}^{M} M_{n+1}-\right.\right.$ $\left.\left.\sum_{j=1, j \neq i}^{n} D_{j}^{M}\right) / 2 A_{i i}^{M}\right\}$; then,

$$
V(t)=\min _{1 \leq i \leq n}\left\{z_{i}(t)\right\} \geq m \text { for any } t>T^{\prime} .
$$

On the other hand, from the $(n+1)$ th equation of $(8)$ and (20), for $t>T^{\prime}+\widehat{\sigma}$, we have

$$
\begin{aligned}
\dot{z}_{n+1}(t) \geq & z_{n+1}(t) \\
& \times\left(-r_{n+1}^{M}+\sum_{j=1}^{n} A_{n+1, j}^{L} m-A_{n+1, n+1}^{M} z_{n+1}\left(t-\sigma_{n+1}\right)\right) .
\end{aligned}
$$

According to the assumption $-r_{n+1}^{M}+\sum_{j=1}^{n} A_{n+1, j}^{L} m>0$, there exists $\bar{m}$ satisfying $\sum_{j=1}^{n} A_{n+1, j}^{L} m-r_{n+1}^{M}-A_{n+1, n+1}^{M} \bar{m}>0$. Let $\lambda=\sum_{j=1}^{n} A_{n+1, j}^{L} m-r_{n+1}^{M}-A_{n+1, n+1}^{M} \bar{m}$. If $z_{n+1}(t) \leq \bar{m}$ for all $t>T^{\prime}+\widehat{\sigma}+\sigma_{n+1}$, then by (21) it follows that $\dot{z}_{n+1}(t) \geq \lambda z_{n+1}(t)$, which leads to a contradiction. Thus, there must exist $T^{\prime \prime} \geq$ $T^{\prime}+\widehat{\sigma}+\sigma_{n+1}$ such that $z_{n+1}\left(T^{\prime \prime}\right)>\bar{m}$. If $z_{n+1}(t)>\bar{m}$ for any $t>T^{\prime \prime}$, then we take $m_{n+1}=\bar{m}$; the conclusions hold. If not, suppose $z_{n+1}(\bar{t}) \leq \bar{m}$, where $\bar{t}>T^{\prime \prime}$; then, from the above discussion, there exist $t^{*}$ and $t^{* *}$ such that $z_{n+1}\left(t^{*}\right)=$ $z_{n+1}\left(t^{* *}\right)=\bar{m}$ and $z_{n+1}(t)<\bar{m}$ for $t^{*}<t<t^{* *}$, where $T^{\prime \prime}<$ $t^{*}<\bar{t}<t^{* *}$. Now suppose that $z_{n+1}(t)$ attains its maximum at $t=\widetilde{t}$; then, $\dot{z}_{n+1}(\widetilde{t})=0$. Equation (21) implies that

$$
-r_{n+1}^{M}+\sum_{j=1}^{n} A_{n+1, j}^{L} m-A_{n+1, n+1}^{M} z_{n+1}\left(\tilde{t}-\sigma_{n+1}\right) \leq 0 .
$$

Equation (22) leads to

$$
z_{n+1}\left(\tilde{t}-\sigma_{n+1}\right) \geq \frac{-r_{n+1}^{M}+\sum_{j=1}^{n} A_{n+1, j}^{L} m}{A_{n+1, n+1}^{M}}>\bar{m} .
$$

From (21) again, we have

$$
\frac{\dot{z}_{n+1}(t)}{z_{n+1}(t)} \geq-r_{n+1}^{M}+\sum_{j=1}^{n} A_{n+1, j}^{L} m-A_{n+1, n+1}^{M} \bar{m} .
$$

Integrating the above inequality from $\tilde{t}-\sigma_{n+1}$ to $\widetilde{t}$, we have

$$
\begin{aligned}
z_{n+1}(\widetilde{t}) & \geq z_{n+1}\left(\widetilde{t}-\sigma_{n+1}\right) e^{\left(-r_{n+1}^{M}+\sum_{j=1}^{n} A_{n+1, j}^{L} m-A_{n+1, n+1}^{M} \bar{m}\right) \sigma_{n+1}} \\
& \geq \bar{m} e^{\left(-r_{n+1}^{M}+\sum_{j=1}^{n} A_{n+1, j}^{L} m-A_{n+1, n+1}^{M} \bar{m}\right) \sigma_{n+1}}>\bar{m} .
\end{aligned}
$$

This contradicts with $z_{n+1}(t)<\bar{m}$ for $t^{*}<t<t^{* *}$. Therefore, $z_{n+1}(t)>\bar{m}$ holds for any $t>T$. Let $m_{n+1}=\bar{m}$; then, $m_{i} \leq$ $z_{i}(t)$ holds for $i=1,2, \ldots, n+1$. In addition, by Lemma 7 , $z_{i}(t) \leq M_{i}(i=1,2, \ldots, n+1)$. Hence, $m_{i} \leq z_{i}(t) \leq M_{i}$ holds for $i=1,2, \ldots, n+1$.This completes the proof.

Theorem 9. In addition to $\left(C_{1}\right)-\left(C_{5}\right)$, further assume that

$\left(C_{6}\right) \min Q_{i}(t)>0, i=1,2, \ldots, n+1$, where

$$
\begin{aligned}
Q_{i}= & A_{i i}(t)-\frac{(n-1) \widetilde{D}_{i}^{M}}{m}-A_{n+1, i}\left(t+\sigma_{i}\right) \\
& -M_{n+1} A_{n+1, i}\left(t+\sigma_{i}\right) \int_{t+\sigma_{i}}^{t+\sigma_{i}+\sigma_{n+1}} A_{n+1, n+1}(s) d s, \quad i \neq j, \\
Q_{i}= & A_{i i}(t)-A_{n+1, i}\left(t+\sigma_{i}\right)-M_{n+1} A_{n+1, i}\left(t+\sigma_{i}\right) \\
& \times \int_{t+\sigma_{i}}^{t+\sigma_{i}+\sigma_{n+1}} A_{n+1, n+1}(s) d s, \quad i=j, i=1,2, \ldots, n, \\
Q_{n+1} & A_{n+1, n+1}(t)-\int_{t}^{t+\sigma_{n+1}} A_{n+1, n+1}(s) d s \\
& \times\left(r_{n+1}(t)+\sum_{j=1}^{n} A_{n+1, j}(t) M_{j}+A_{n+1, n+1}(t) M_{n+1}\right) \\
& -A_{n+1, n+1}\left(t+\sigma_{n+1}\right) M_{n+1} \\
& \times \int_{t+\sigma_{n+1}}^{t+2 \sigma_{n+1}} A_{n+1, n+1}(s) d s-\sum_{i=1}^{n} A_{i, n+1}(t) .
\end{aligned}
$$

Then, system (1) has one w-periodic solution which is globally asymptotically stable.

Proof. By Lemmas 3 and 6, we know that (8) has one $\omega$-periodic solution. Hence, we only need to show the global asymptotic stability of the positive periodic solution of (8). Let $x^{*}(t)=\left(x_{1}^{*}(t), x_{2}^{*}(t), \ldots, x_{n+1}^{*}(t)\right)^{T}$ be a positive $\omega$-periodic solution of (1) and let $x(t)=\left(x_{1}(t)\right.$, $\left.x_{2}(t), \ldots, x_{n+1}(t)\right)^{T}$ be any positive solution of (1); then, $z^{*}(t)=\left(z_{1}^{*}(t), z_{2}^{*}(t), \ldots, z_{n+1}^{*}(t)\right)^{T}, z_{i}^{*}(t)=\prod_{0<t_{k}<t}\left(1-c_{i k}\right)^{-1}$ $x_{i}^{*}(t)(i=1,2, \ldots, n+1)$ is the positive $\omega$-periodic solution of 
(8) and $z(t)=\left(z_{1}(t), z_{2}(t), \ldots, z_{n+1}(t)\right)^{T}, z_{i}(t)=\prod_{0<t_{k}<t}(1-$ $\left.c_{i k}\right)^{-1} x_{i}(t)(i=1,2, \ldots, n+1)$ is the positive solution of $(8)$.

From Theorem 8, there exists $T>0$, for all $t>T, m_{i}<$ $z_{i}(t) \leq M_{i}, m_{i}<z_{i}^{*}(t) \leq M_{i}, i=1,2, \ldots, n+1$. Consider the following Lyapunov functional:

$$
V_{1}(t)=\sum_{i=1}^{n}\left|\ln z_{i}(t)-\ln z_{i}^{*}(t)\right|
$$

Calculate and estimate the upper right derivative of $V_{1}(t)$ along the solution of (8); then,

$$
\begin{aligned}
& D^{+} V_{1}(t)=\sum_{i=1}^{n} \operatorname{sgn}\left(z_{i}^{*}(t)-z_{i}(t)\right)\left(\frac{\dot{z}_{i}^{*}(t)}{z_{i}^{*}(t)}-\frac{\dot{z}_{i}(t)}{z_{i}(t)}\right) \\
& =\sum_{i=1}^{n} \operatorname{sgn}\left(z_{i}^{*}(t)-z_{i}(t)\right) \\
& \times\left(-A_{i i}(t)\left(z_{i}^{*}(t)-z_{i}(t)\right)\right. \\
& \left.-A_{i, n+1}(t)\left(z_{n+1}^{*}(t)-z_{n+1}(t)\right)\right) \\
& +\sum_{i=1}^{n} \operatorname{sgn}\left(z_{i}^{*}(t)-z_{i}(t)\right) \\
& \times \sum_{j=1, j \neq i}^{n} \widetilde{D}_{j}(t)\left(\frac{z_{j}^{*}(t)}{z_{i}^{*}(t)}-\frac{z_{j}(t)}{z_{i}(t)}\right) \\
& \leq \sum_{i=1}^{n}\left(-A_{i i}(t)\left|z_{i}^{*}(t)-z_{i}(t)\right|\right. \\
& +A_{i, n+1}(t)\left|z_{n+1}^{*}(t)-z_{n+1}(t)\right| \\
& \left.+\sum_{j=1, j \neq i}^{n} \bar{D}_{j}(t)\right)
\end{aligned}
$$

where

$$
\bar{D}_{j}(t)= \begin{cases}\widetilde{D}_{j}(t)\left(\frac{z_{j}^{*}(t)}{z_{i}^{*}(t)}-\frac{z_{j}(t)}{z_{i}(t)}\right), & z_{i}^{*}(t)>z_{i}(t) \\ \widetilde{D}_{j}(t)\left(\frac{z_{j}(t)}{z_{i}(t)}-\frac{z_{j}^{*}(t)}{z_{i}^{*}(t)}\right), & z_{i}^{*}(t)<z_{i}(t) .\end{cases}
$$

By using similar analysis in [12], we have the following.

(i) If $z_{i}^{*}(t)>z_{i}(t)$, then

$$
\bar{D}_{j}(t) \leq \frac{\widetilde{D}_{j}(t)}{z_{i}^{*}(t)}\left(z_{j}^{*}(t)-z_{j}(t)\right) \leq \frac{\widetilde{D}_{j}^{M}}{m}\left|z_{j}^{*}(t)-z_{j}(t)\right| .
$$

(ii) If $z_{i}^{*}(t)<z_{i}(t)$, then

$$
\bar{D}_{j}(t) \leq \frac{\widetilde{D}_{j}(t)}{z_{i}(t)}\left(z_{j}(t)-z_{j}^{*}(t)\right) \leq \frac{\widetilde{D}_{j}^{M}}{m}\left|z_{j}^{*}(t)-z_{j}(t)\right| .
$$

Therefore, for $t>T$,

$$
\begin{aligned}
D^{+} V_{1}(t) \leq \sum_{i=1}^{n}( & A_{i i}(t)\left|z_{i}^{*}(t)-z_{i}(t)\right| \\
& +A_{i, n+1}(t)\left|z_{n+1}^{*}(t)-z_{n+1}(t)\right| \\
& \left.+\sum_{j=1, j \neq i}^{n} \widetilde{D}_{j}^{M} \frac{1}{m}\left|z_{j}^{*}(t)-z_{j}(t)\right|\right) .
\end{aligned}
$$

Define $V_{2}(t)=\left|\ln z_{n+1}^{*}(t)-\ln z_{n+1}(t)\right|$. Calculating the upper right derivative of $V_{2}(t)$ along the solution of system (8), for $t>T+\sigma$, we have

$$
\begin{aligned}
& D^{+} V_{2}(t)=\operatorname{sgn}\left(z_{n+1}^{*}(t)-z_{n+1}(t)\right) \\
& \times\left(\frac{\dot{z}_{n+1}^{*}(t)}{z_{n+1}^{*}(t)}-\frac{\dot{z}_{n+1}(t)}{z_{n+1}(t)}\right) \\
& =\operatorname{sgn}\left(z_{n+1}^{*}(t)-z_{n+1}(t)\right) \\
& \times\left\{-A_{n+1, n+1}(t)\right. \\
& \times\left(z_{n+1}^{*}\left(t-\sigma_{n+1}\right)-z_{n+1}\left(t-\sigma_{n+1}\right)\right) \\
& +\sum_{j=1}^{n} A_{n+1, j}(t)\left(z_{j}^{*}\left(t-\sigma_{j}\right)\right. \\
& \left.\left.-z_{j}\left(t-\sigma_{j}\right)\right)\right\} \\
& =\operatorname{sgn}\left(z_{n+1}^{*}(t)-z_{n+1}(t)\right) \\
& \times\left\{-A_{n+1, n+1}(t)\left(z_{n+1}^{*}(t)-z_{n+1}(t)\right)\right. \\
& +\sum_{j=1}^{n} A_{n+1, j}(t) \times\left(z_{j}^{*}\left(t-\sigma_{j}\right)\right. \\
& \left.-z_{j}\left(t-\sigma_{j}\right)\right) \\
& +A_{n+1, n+1}(t) \\
& \left.\times \int_{t-\sigma_{n+1}}^{t}\left(\dot{z}_{n+1}^{*}(u)-\dot{z}_{n+1}(u)\right) d u\right\} \\
& =\operatorname{sgn}\left(z_{n+1}^{*}(t)-z_{n+1}(t)\right) \\
& \times\left\{-A_{n+1, n+1}(t)\left(z_{n+1}^{*}(t)-z_{n+1}(t)\right)\right. \\
& +\sum_{j=1}^{n} A_{n+1, j}(t)
\end{aligned}
$$




$$
\begin{aligned}
& \times\left(z_{j}^{*}\left(t-\sigma_{j}\right)-z_{j}\left(t-\sigma_{j}\right)\right) \\
& +A_{n+1, n+1}(t) \\
& \times \int_{t-\sigma_{n+1}}^{t}\left[\left(-r_{n+1}(u)\right.\right. \\
& +\sum_{j=1}^{n} A_{n+1, j}(u) z_{j}\left(u-\sigma_{j}\right) \\
& \left.-A_{n+1, n+1}(u) z_{n+1}\left(u-\sigma_{n+1}\right)\right) \\
& \times\left(z_{n+1}^{*}(u)-z_{n+1}(u)\right) \\
& -A_{n+1, n+1}(u) z_{n+1}^{*}(u) \\
& \times\left(z_{n+1}^{*}\left(u-\sigma_{n+1}\right)\right. \\
& \left.-z_{n+1}\left(u-\sigma_{n+1}\right)\right) \\
& +\sum_{j=1}^{n} A_{n+1, j}(u) z_{n+1}^{*}(u) \\
& \times\left(z_{j}^{*}\left(u-\sigma_{j}\right)\right. \\
& \left.\left.\left.-z_{j}\left(u-\sigma_{j}\right)\right)\right] d u\right\} .
\end{aligned}
$$

According to (14) and the above equality, for $t \geq T+\sigma$, we have

$$
\begin{aligned}
& D^{+} V_{2}(t) \leq-A_{n+1, n+1}(t)\left|z_{n+1}^{*}(t)-z_{n+1}(t)\right| \\
&+\sum_{j=1}^{n} A_{n+1, j}(t)\left|z_{j}^{*}\left(t-\sigma_{j}\right)-z_{j}\left(t-\sigma_{j}\right)\right| \\
&+A_{n+1, n+1}(t) \\
& \times \int_{t-\sigma_{n+1}}^{t}\left[\left(r_{n+1}(u)+\sum_{j=1}^{n} A_{n+1, j}(u) M\right.\right. \\
&\left.+A_{n+1, n+1}(u) M_{n+1}\right) \\
& \times\left|z_{n+1}^{*}(u)-z_{n+1}(u)\right| \\
&+ A_{n+1, n+1}(u) M_{n+1} \\
& \times \mid z_{n+1}^{*}\left(u-\sigma_{n+1}\right) \\
&-z_{n+1}\left(u-\sigma_{n+1}\right) \mid
\end{aligned}
$$

$$
\begin{aligned}
& +\sum_{j=1}^{n} A_{n+1, j}(u) M_{n+1} \\
& \left.\times\left|z_{j}^{*}\left(u-\sigma_{j}\right)-z_{j}\left(u-\sigma_{j}\right)\right|\right] d u .
\end{aligned}
$$

Define

$$
\begin{aligned}
& V_{3}(t)=\sum_{j=1}^{n} \int_{t-\sigma_{j}}^{t} A_{n+1, j}\left(s+\sigma_{j}\right)\left|z_{j}^{*}(s)-z_{j}(s)\right| \\
& +\int_{t}^{t+\sigma_{n+1}} \int_{s-\sigma_{n+1}}^{t} A_{n+1, n+1}(s) \\
& \times\left\{\left(r_{n+1}(u)\right.\right. \\
& +\sum_{j=1}^{n} A_{n+1, j}(u) M \\
& \left.+A_{n+1, n+1}(u) M_{n+1}\right) \\
& \times\left|z_{n+1}^{*}(u)-z_{n+1}(u)\right| \\
& +A_{n+1, n+1}(u) M_{n+1} \\
& \times \mid z_{n+1}^{*}\left(u-\sigma_{n+1}\right) \\
& -z_{n+1}\left(u-\sigma_{n+1}\right) \mid \\
& +\sum_{j=1}^{n} A_{n+1, j}(u) M_{n+1} \\
& \times \mid z_{j}^{*}\left(u-\sigma_{j}\right) \\
& \left.-z_{j}\left(u-\sigma_{j}\right) \mid\right\} d u d s .
\end{aligned}
$$

From (34) and (35), for $t \geq T+\sigma$, then

$$
\begin{aligned}
D^{+} V_{2}(t)+V_{3}^{\prime}(t) \leq & -A_{n+1, n+1}(t) \\
& \times\left|z_{n+1}^{*}(t)-z_{n+1}(t)\right| \\
& +\sum_{j=1}^{n} A_{n+1, j}\left(t+\sigma_{j}\right)\left|z_{j}^{*}(t)-z_{j}(t)\right| \\
& +\int_{t}^{t+\sigma_{n+1}} A_{n+1, n+1}(s) d s \\
& \times\left[\left(r_{n+1}(t)+\sum_{j=1}^{n} A_{n+1, j}(t) M\right.\right.
\end{aligned}
$$




$$
\begin{aligned}
& \left.+A_{n+1, n+1}(t) M_{n+1}\right) \\
& \times\left|z_{n+1}^{*}(t)-z_{n+1}(t)\right| \\
& +A_{n+1, n+1}(t) M_{n+1} \\
& \times \mid z_{n+1}^{*}\left(t-\sigma_{n+1}\right) \\
& \quad-z_{n+1}\left(t-\sigma_{n+1}\right) \mid \\
& +\sum_{j=1}^{n} A_{n+1, j}(t) M_{n+1} \\
& \left.\times\left|z_{j}^{*}\left(t-\sigma_{j}\right)-z_{j}\left(t-\sigma_{j}\right)\right|\right] .
\end{aligned}
$$

Define

$$
\begin{gathered}
V_{4}(t)=\int_{t-\sigma_{j}}^{t} \int_{u+\sigma_{j}}^{u+\sigma_{j}+\sigma_{n+1}} M_{n+1} A_{n+1, n+1}(s) \\
\quad \times \sum_{j=1}^{n} A_{n+1, j}\left(u+\sigma_{j}\right) \\
\times\left|z_{j}^{*}(u)-z_{j}(u)\right| d s d u \\
+M_{n+1} \int_{t-\sigma_{n+1}}^{t} \int_{u+\sigma_{n+1}}^{u+2 \sigma_{n+1}} A_{n+1, n+1}(s) A_{n+1, n+1} \\
\times\left(u+\sigma_{n+1}\right) \\
\times\left|z_{n+1}^{*}(u)-z_{n+1}(u)\right| d s d u .
\end{gathered}
$$

For $t \geq T+\sigma$, from (36) and (37), then

$$
\begin{aligned}
D^{+} & \left(V_{2}+V_{3}+V_{4}\right) \\
\leq & -A_{n+1, n+1}(t)\left|z_{n+1}^{*}(t)-z_{n+1}(t)\right| \\
& +\sum_{j=1}^{n} A_{n+1, j}\left(t+\sigma_{j}\right) \\
& \times\left|z_{j}^{*}(t)-z_{j}(t)\right|+\int_{t}^{t+\sigma_{n+1}} A_{n+1, n+1}(s) d s \\
& \times\left(r_{n+1}(t)+\sum_{j=1}^{n} A_{n+1, j}(t) M_{+} A_{n+1, n+1}(t) M_{n+1}\right) \\
& \times\left|z_{n+1}^{*}(t)-z_{n+1}(t)\right| \\
& +M_{n+1} \sum_{j=1}^{n} A_{n+1, j}\left(t+\sigma_{j}\right) \int_{t+\sigma_{j}}^{t+\sigma_{j}+\sigma_{n+1}} A_{n+1, n+1}(s) d s
\end{aligned}
$$

$$
\begin{aligned}
& \times\left|z_{j}^{*}(t)-z_{j}(t)\right|+A_{n+1, n+1}\left(t+\sigma_{n+1}\right) M_{n+1} \\
& \times \int_{t+\sigma_{n+1}}^{t+2 \sigma_{n+1}} A_{n+1, n+1}(s) d s\left|z_{n+1}^{*}(t)-z_{n+1}(t)\right| .
\end{aligned}
$$
that

Let $V(t)=\sum_{i=1}^{4} V_{i}(t)$; then, it follows from (18) and (32)

$$
D^{+} V(t) \leq-\sum_{i=1}^{n+1} Q_{i}(t)\left|z_{i}^{*}(t)-z_{i}(t)\right|, \quad t \geq T+\sigma,
$$

where $Q_{i}(t)$ are defined by (26).

According to $\left(C_{6}\right)$, there exist $\alpha_{i}>0(i=1,2, \ldots, n+1)$ and $T^{*}>T+\sigma$ such that $Q_{i}(t) \geq \alpha_{i}>0$ for $t \geq T^{*}$. Integrating (39) on interval $\left[T^{*}, t\right]$ yields

$$
V(t)+\sum_{i=1}^{n+1} \int_{T^{*}}^{t} Q_{i}(s)\left|z_{i}^{*}(s)-z_{i}(t)\right| d s \leq V\left(T^{*}\right) .
$$

It follows from (39) and (40) that

$$
\sum_{i=1}^{n+1} \int_{T^{*}}^{t} \alpha_{i}(s)\left|z_{i}^{*}(s)-z_{i}(t)\right| d s \leq V\left(T^{*}\right)<\infty, \quad t \geq T^{*} .
$$

Since $\dot{z}_{i}^{*}(t), \dot{z}_{i}(t)(i=1,2, \ldots, n+1)$ are bounded for $t \geq$ $T^{*}$, then $\left|z_{i}^{*}(t)-z_{i}(t)\right|$ is uniformly continuous on $\left[T^{*}, \infty\right)$. By Lemma $5, \lim _{t \rightarrow \infty} \sum_{i=1}^{n+1}\left|z_{i}^{*}(t)-z_{i}(t)\right|=0$. Therefore, $\lim _{t \rightarrow \infty}\left|z_{i}^{*}(t)-z_{i}(t)\right|=0$; that is, $\lim _{t \rightarrow \infty}\left|x_{i}^{*}(t)-x_{i}(t)\right|=0$, $i=1,2, \ldots, n+1$. This completes the proof.

\section{Example and Simulations}

In this section, an illustrative example and numerical analysis are given to show the effectiveness of the main results.

Example 1. Consider the following dispersed predator-prey system with delays and impulses:

$$
\begin{gathered}
\dot{x}_{1}(t)=x_{1}(t)\left(r_{1}(t)-a_{11}(t) x_{1}(t)\right. \\
\left.\quad-a_{13}(t) x_{3}(t)\right)+D_{2}(t)\left(x_{2}(t)-x_{1}(t)\right), \\
\begin{array}{r}
\dot{x}_{2}(t)=x_{2}(t)\left(r_{2}(t)-a_{22}(t) x_{2}(t)\right. \\
\left.\quad-a_{23}(t) x_{3}(t)\right)+D_{1}(t)\left(x_{1}(t)-x_{2}(t)\right), \\
\dot{x}_{3}(t)=x_{3}(t)\left(-r_{3}(t)+a_{31}(t) x_{1}\left(t-\sigma_{1}\right)\right. \\
\left.\quad+a_{32}(t) x_{2}\left(t-\sigma_{2}\right)-a_{33}(t) x_{3}\left(t-\sigma_{3}\right)\right), \\
\Delta x_{i}\left(t_{k}\right)=-c_{i k} x_{i}\left(t_{k}\right), \quad i=1,2,3, \quad k=1,2, \ldots \\
\quad t \neq t_{k} .
\end{array}
\end{gathered}
$$

Take $r_{1}=r_{2}=2, r_{3}=3, a_{11}(t)=3 / 2+\sin t / 2, a_{22}=2$, $a_{13}(t)=a_{23}(t)=1, a_{31}(t)=7 / 4+\sin t / 4, a_{32}(t)=15+$ 


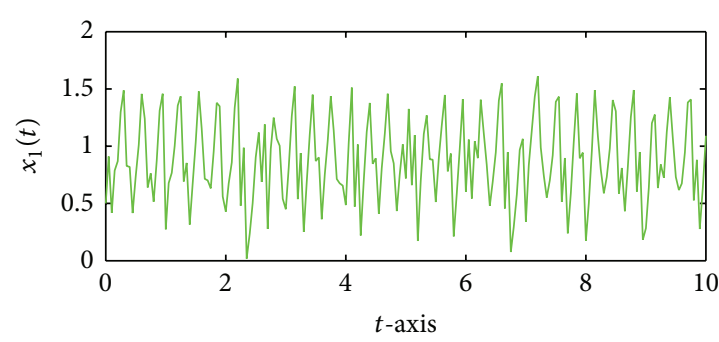

(a)

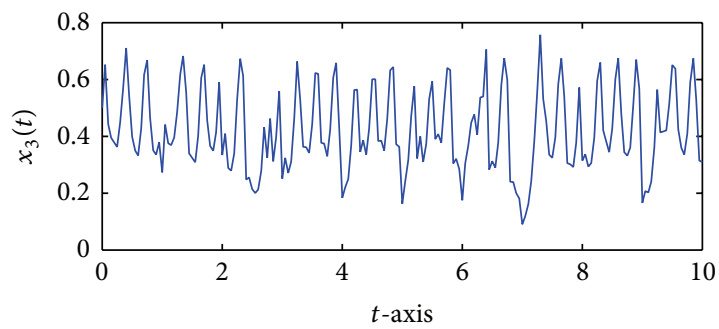

(c)

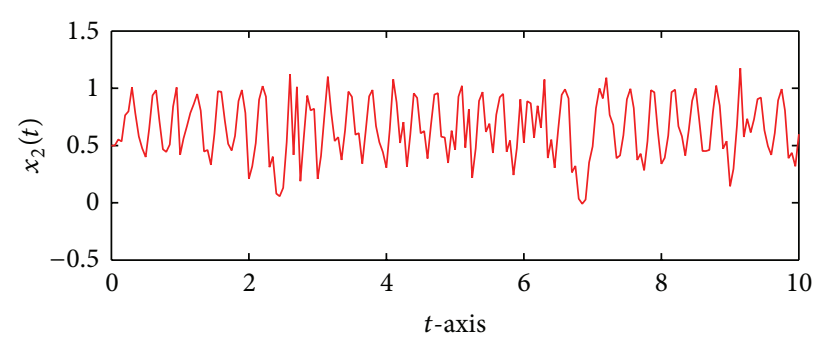

(b)

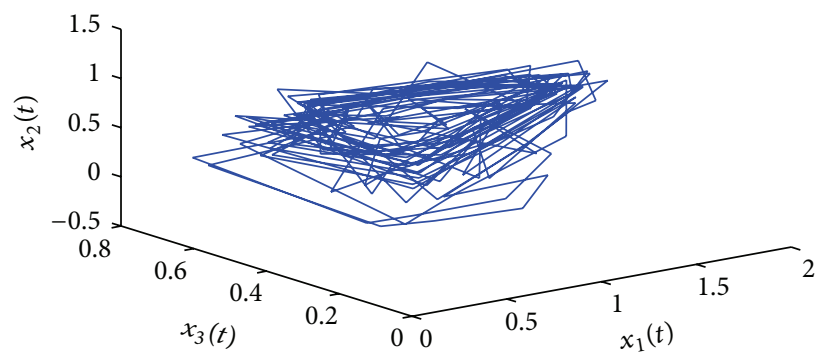

(d)

FIgure 1: Dynamics of (42). (a) Time series of $x_{1}$, (b) time series of $x_{2}$, (c) time series of $x_{3}$, and (d) portrait of $\left(x_{1}, x_{2}, x_{3}\right)$.

$3 \cos t, a_{33}(t)=31-\sin t, D_{1}(t)=D_{2}(t)=1 / 2, \sigma_{1}=\sigma_{2}=$ $0.2, \sigma_{3}=0, c_{i k}=1 / 2$. Then, $\omega=\pi$. Let $\left\{t_{k}, k=1,2, \ldots\right\} \cap$ $[0, \omega]=\left\{t_{1}, t_{2}\right\}$. By calculating, it is not difficult to show that $\left(C_{3}\right)-\left(C_{5}\right)$ hold. Hence, from Lemma 3 and Theorem 8 , system (42) has at least one positive $\pi$-periodic solution and (42) is persistent. By numerical analysis, the existence and persistence of (42) can be showed clearly; see Figure 1.

\section{Conclusion}

For predator-prey system (1), the periodic solution is studied by authors [3], but in the real world, in addition to periodic behavior, a hallmark of observed population densities is their permanent and stable behaviors. Then, it is necessary and valuable to investigate the stability of (1). In this paper, by constructing a suitable Lyapunov functional, we are devoted to the study of global attractivity of positive periodic solution of (1). Further, an example and numerical analysis are given to show the effectiveness of the main results. In addition, the existence of almost periodic solution is interesting and will be our research work in the future.

\section{Conflict of Interests}

The authors declare that there is no conflict of interests regarding the publication of this paper.

\section{Acknowledgments}

The authors would like to thank the referees for their very suggestive comments. This paper is supported by the Natural Science Foundation of Guangxi (2013GXNSFAA019003) and National Natural Science Foundation of China (11161015, 11361012 , and 11161011) and partially supported by the National
High Technology Research and Development Program 863 under Grant no. 2013AA12A402.

\section{References}

[1] Y. K. Li and L. L. Zhao, "Positive periodic solutions for a neutral Lotka-Volterra system with state dependent delays," Communications in Nonlinear Science and Numerical Simulation, vol. 14, no. 4, pp. 1561-1569, 2009.

[2] X. Meng and L. Chen, "Periodic solution and almost periodic solution for a nonautonomous Lotka-Volterra dispersal system with infinite delay," Journal of Mathematical Analysis and Applications, vol. 339, no. 1, pp. 125-145, 2008.

[3] Q. Liu and S. Dong, "Periodic solutions for a delayed predatorprey system with dispersal and impulses," Electronic Journal of Differential Equations, vol. 31, pp. 1-14, 2005.

[4] Y. F. Shao, B. X. Dai, and Z. G. Luo, "The dynamics of an impulsive one-prey multi-predators system with delay and Holling-type II functional response," Applied Mathematics and Computation, vol. 217, no. 6, pp. 2414-2424, 2010.

[5] S. Ahmad and I. M. Stamova, "Asymptotic stability of competitive systems with delays and impulsive perturbations," Journal of Mathematical Analysis and Applications, vol. 334, no. 1, pp. 686-700, 2007.

[6] R. Liang and J. Shen, "Uniform stability theorems for delay differential equations with impulses," Journal of Mathematical Analysis and Applications, vol. 326, no. 1, pp. 62-74, 2007.

[7] G. Wei and J. Shen, "Asymptotic behavior of solutions of nonlinear impulsive delay differential equations with positive and negative coefficients," Mathematical and Computer Modelling, vol. 44, no. 11-12, pp. 1089-1096, 2006.

[8] R. Xu and Z. Ma, "The effect of dispersal on the permanence of a predator-prey system with time delay," Nonlinear Analysis. Real World Applications, vol. 9, no. 2, pp. 354-369, 2008. 
[9] I. Barbalat, "Systems d'equations differential doscillations nonlinearies," Revue Roumaine de Mathématique Pures et Appliquées, vol. 4, pp. 267-270, 1959.

[10] J. Yan and A. Zhao, "Oscillation and stability of linear impulsive delay differential equations," Journal of Mathematical Analysis and Applications, vol. 227, no. 1, pp. 187-194, 1998.

[11] W. Wendi and Z. Ma, "Harmless delays for uniform persistence," Journal of Mathematical Analysis and Applications, vol. 158, no. 1, pp. 256-268, 1991.

[12] F. Chen, "Positive periodic solutions of neutral Lotka-Volterra system with feedback control," Applied Mathematics and Computation, vol. 162, no. 3, pp. 1279-1302, 2005. 


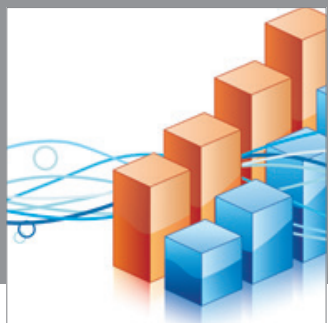

Advances in

Operations Research

mansans

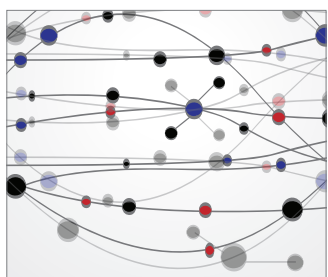

The Scientific World Journal
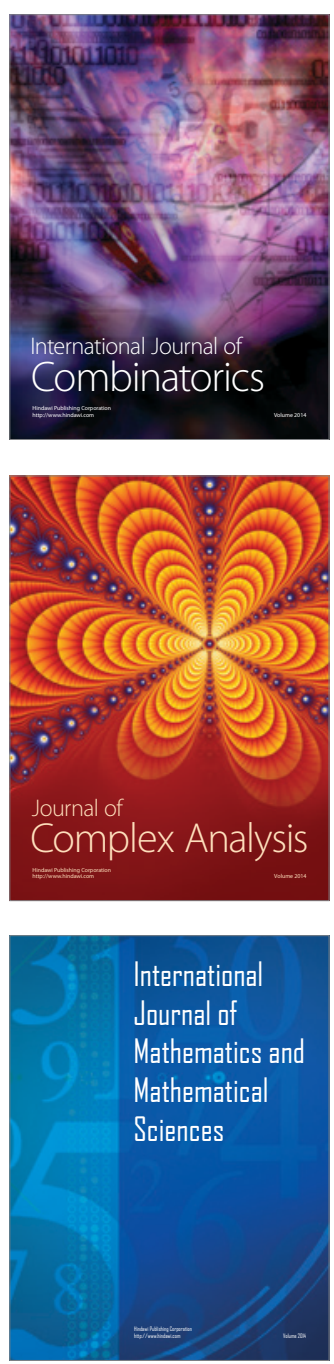
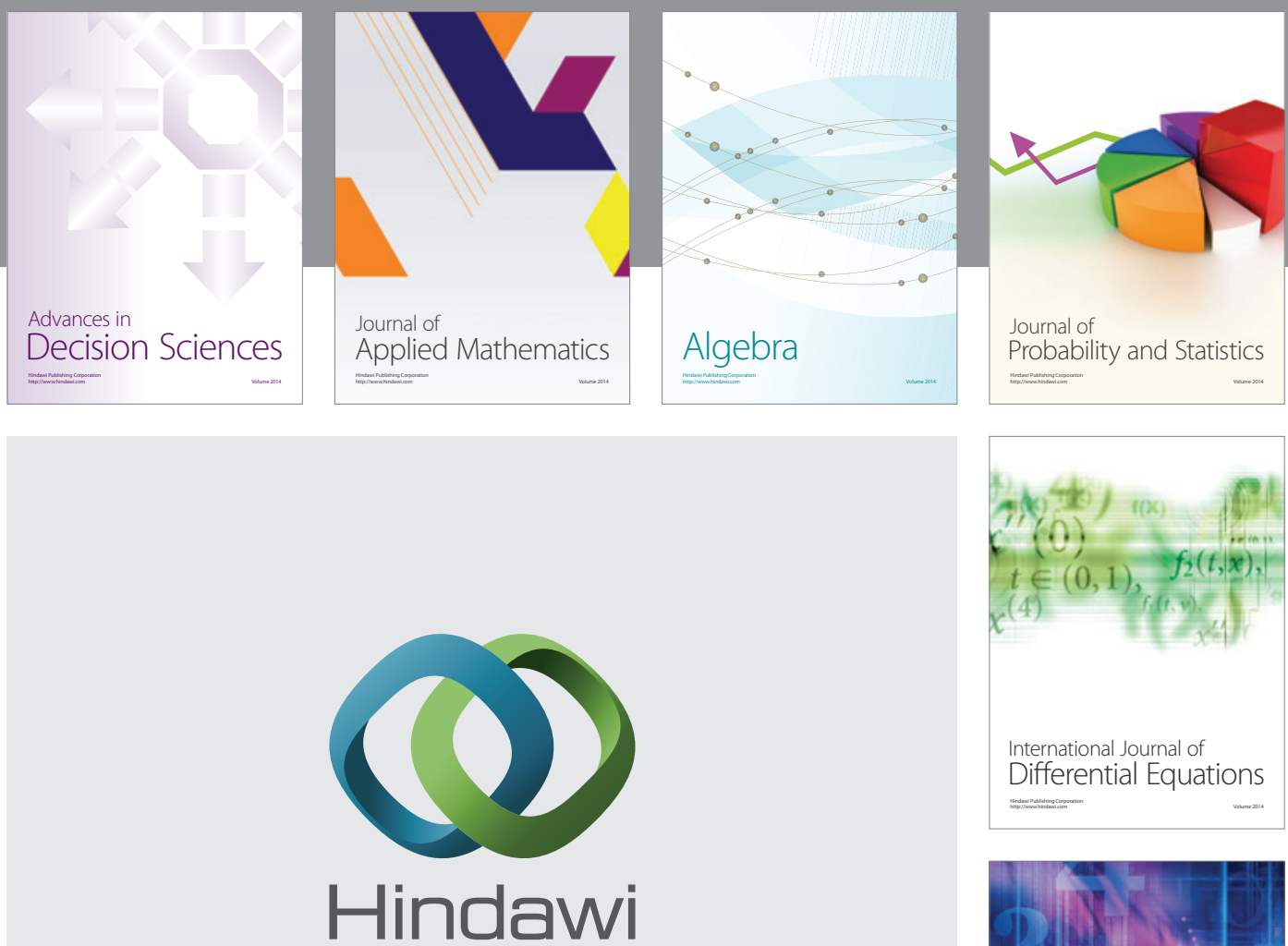

Submit your manuscripts at http://www.hindawi.com
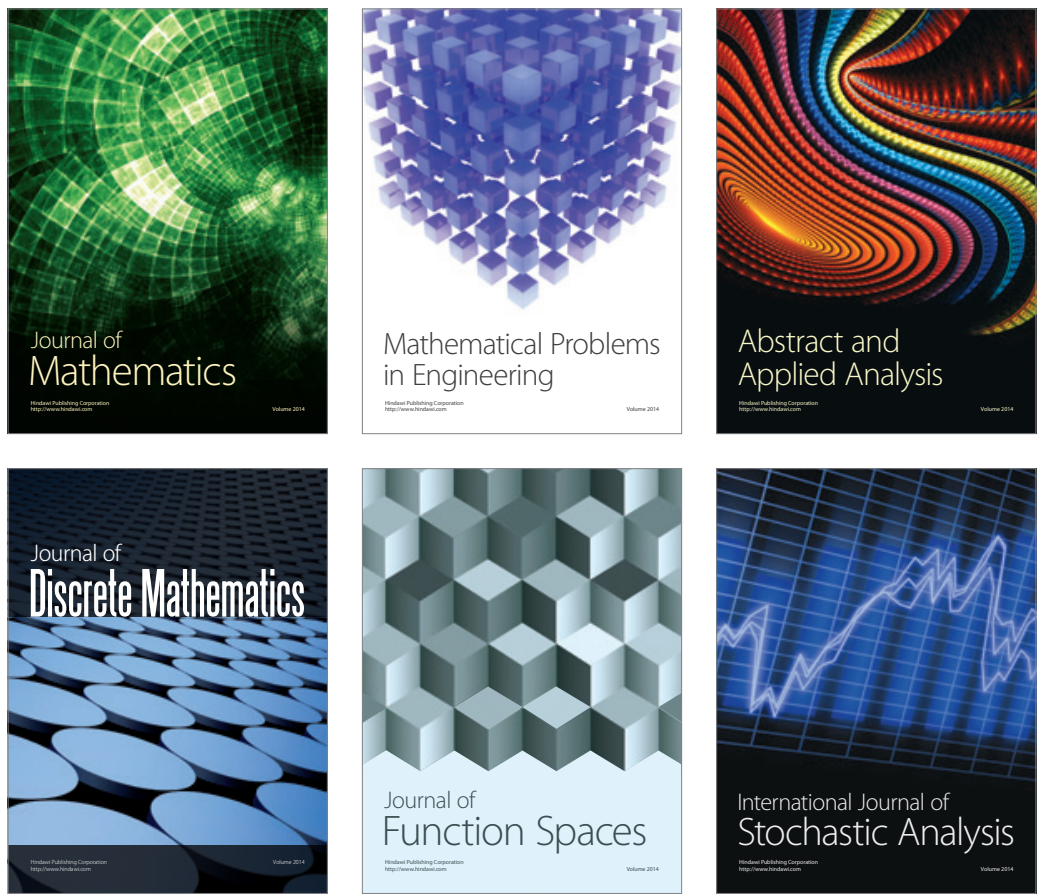

Journal of

Function Spaces

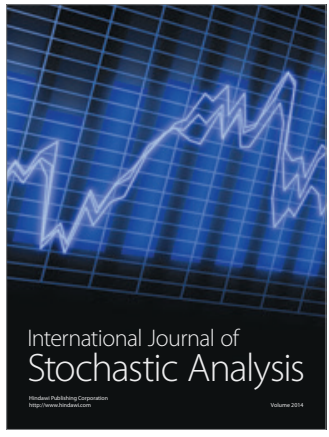

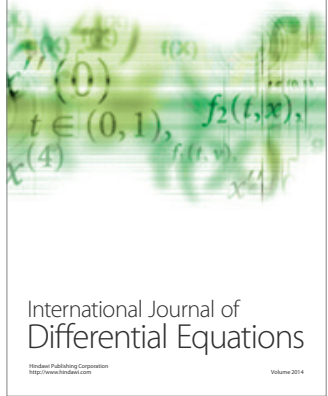
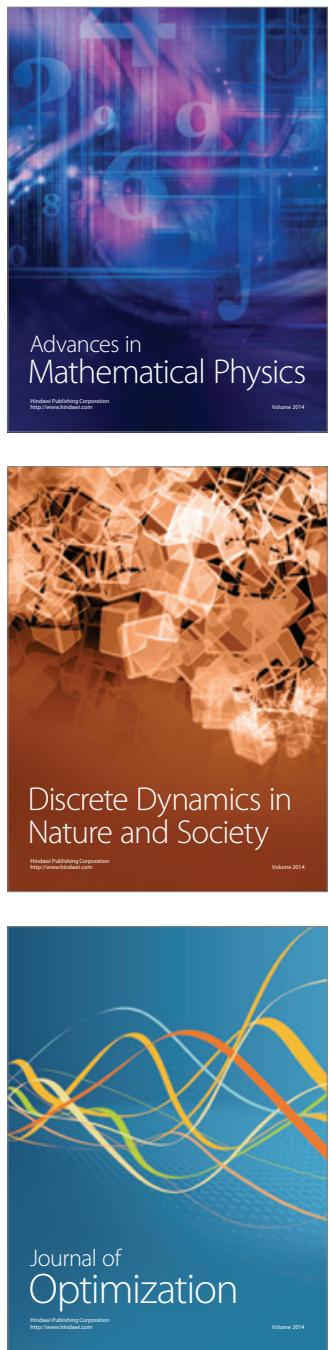